\title{
The Role of EGFR, Hepatocyte Growth Factor Receptor (c-Met), c-ErbB2 (HER2-neu) and Clinicopathological Parameters in the Pathogenesis and Prognosis of Chordoma
}

\author{
Zeynep TOSUNER', Süheyla UYAR BOZKURT², Türker KILIÇ³, Baran YILMAZ³ \\ Department of Pathology, 'Bezmialem Vakif University Faculty of Medicine, iSTANBUL, TURKEY, ${ }^{2}$ Marmara University School of Medicine, iSTANBUL, TURKEY \\ ${ }^{3}$ Department of Neurosurgery, Bahcesehir University Faculty of Medicine, iSTANBUL, TURKEY
}

\begin{abstract}
Objective: Chordoma is a rare malignant bone tumor with a poor outcome. Although radiotherapy and gamma knife surgery have been used for treatment, providing a cure for the tumor is not easy, because of the frequent recurrences. Molecular targeted therapy against tyrosine kinases has been effective in the treatment of malignancies such as breast and lung cancers and brain tumors. We aimed to analyse the histopathological features of chordomas and the immunoexpression profiles of the three receptor tyrosine kinases of EGFR, c-Met and c-Erb-B2 in chordomas. We have correlated these results with recurrence and overall survival status of the patients.
\end{abstract}

Material and Method: We studied 49 chordoma patients in order to evaluate the histopathological features and immunohistochemical stainings by EGFR, c-Met and c-ErbB2 antibodies. Of the 49 patients, follow up data was available for 40 patients. Clinical data of the patients were correlated with histopathological features and survival analysis was performed.

Results: The immunostaining rate by EGFR and c-Met was $73.5 \%$ and $12.2 \%$ respectively. None of the cases showed immunoreactivity by c-ErbB2 (0\%). Of the 40 cases, 17 cases showed recurrences. EGFR expression was detected in 14 recurrent (14/17) and 17 non-recurrent cases (17/23). Four of the 17 recurrent cases (4/17) were positive by c-Met, while none of the non-recurrent cases $(0 / 23)$ were positive by this antibody. Significantly, all cases with positive c-Met expression showed recurrences $(\mathrm{p}<0.05)$.

Conclusion: Our study indicates that EGFR expression is detected in the majority of chordoma cases. c-Met expression can be used as a prognostic indicator for chordoma.

Key Words: Chordoma, EGFR, c-Met, c-Erb B2

\section{INTRODUCTION}

Chordoma is a rare malignant bone tumor originating from notochordal remnants $(1,2)$. The complete resection of this tumor is usually not available because of its invasiveness to adjacent structures. Although radiotherapy and gamma knife surgery have been tried for treatment, local control cannot be provided in most of the cases due to the extensive recurrence ability of the tumor (3). There are few studies investigating the effect of treatment modalities on the survival and prognosis of chordoma patients $(3,4)$. Histopathological properties of this tumor such as mitosis and necrosis have been analysed in a number of studies in order to understand their relations with the prognosis and survival. However limited information is available from these studies and also the sample sizes are small because of the rare incidence of chordoma. There are also discordances

(Turk Patoloji Derg 2017, 33:112-120)

Received : 23.03.2016 Accepted : 24.10.2016 between the results of different studies. Despite several proposed prognostic parameters, no evidence-based parameter has been defined to predict survival (5-7)

Receptor tyrosine kinases (RTKs), besides being the key regulators of cell processes including cell growth and angiogenesis, have critical roles in the development and progression of tumors.

The epidermal growth factor receptor (EGFR), ErbB2 (HER-2 neu) and c-met are the members of ErbB family of RTKs. These trans-membrane proteins are activated by binding with peptide growth factors of the EGFfamily of proteins. EGFR and its three related proteins (the ErbB family) play essential roles in both normal physiological conditions and malignancies. Upon binding its ligands, dynamic conformational changes occur in both extracellular and intracellular domains of the receptor

Correspondence: Zeynep TOSUNER

Bezmialem Vakıf University, Faculty of Medicine,

Department of Pathology, İSTANBUL, TURKEY

E-mail: zeyneptosuner@yahoo.com.tr Phone: +90 5327366951 
tyrosine kinases, resulting in the transphosphorylation of tyrosine residues in the C-terminal regulatory domain. These provide docking sites for downstream molecules and lead to the evasion of apoptosis, and to proliferation, invasion and metastases. Mutation in the tyrosine kinase domain of the EGFR gene was found in a subset of lung cancers (8).

ErbB2 activation also occurs in a ligand-independent manner, particularly when the receptor is found to be mutated or overexpressed (9). Amplification and overexpression of the proto-oncogene ErbB2 are found in approximately 15 to $20 \%$ of all invasive breast cancers (10).

The c-Met proto-oncogene is located on chromosome 7q2131 (11). The ligand for c-Met is hepatocyte growth factor (HGF), also known as scatter factor (SF) (12). HGF acts as a cytokine, promoting cell proliferation, survival, motility, scattering, differentiation and morphogenesis (13). Recent studies have revealed that the molecular treatments against EGFR, ErbB2 and c-met succeed in cases of lung and breast carcinomas and glioblastomas, respectively $(14,15)$. Based on the molecular biology of chordomas, targeted therapy alternatives have been developed (16-18). However, only a few case reports are found in the literature regarding this issue.

Chordomas show dual expression of epithelial and mesenchymal proteins (2). EGFR, c-Met and c-ErbB2 overexpressions have been demonstrated in a majority of malignant tumors $(14,15,19-21)$. Receptor tyrosine kinases are the novel candidate molecular markers to shed light on the pathogenesis of chordoma.
The aim of this study was the evaluation of histopathological properties such as tumor, type, mitosis, necrosis pleomorphism, hyperchromatic nuclei, apoptosis and presence of mucin, and the immunoexpression patterns of EGFR, c-Met and c-Erb-B2, in order to understand their role in the pathogenesis, prognosis, recurrence and survival rates of chordoma.

\section{MATERIAL and METHODS}

Forty-nine skull base and non-skull base chordomas were included in our study in order to analyze the expressions of EGFR (c-ErbB1), ErbB2 and c-Met by the streptavidinbiotin horseradish peroxidase method (Table I). The expression levels of these antibodies and their correlations with the histopathological properties of chordoma such as pleomorphism, mitosis, necrosis, hyperchromasia, extracellular mucin deposition and apoptosis (Table II) were evaluated together with the clinical follow-up data including the patient's age, sex, recurrences and overall survival status.

Clinical information and follow-up data were obtained from the Neurosurgery Department and telephone interviews of the patients or family members were conducted. Recurrence was defined as the relapse of disease that occurred more than 6 months after the initial surgery. Overall survival data was dependent on the current status of the patients as being dead or alive. Since autopsies were not performed in cases of death, disease-dependent death interpretation was not available.

All Hematoxylin \& Eosin (H\&E)-stained slides were reviewed by two pathologists for the confirmation of the

Table I: Characteristics of patients with chordoma

\begin{tabular}{lcccc}
\hline & Conventional & Chondroid & Dedifferentiated & Total \\
\hline Number of Patients & 40 & 8 & 1 & 49 \\
\hline Sex & & & & 0 \\
\hline$\quad$ Male & 26 & 5 & 1 & 31 \\
\hline$\quad$ Female & 14 & 3 & 53 & 41 \\
\hline Age (years) & 41 & 40 & & 17 \\
\hline$\quad$ Median & & & 0 & 23 \\
\hline Recurrence status & 15 & 2 & 1 & 9 \\
\hline$\quad$ Recurrence positive & 16 & 6 & 0 & 40 \\
\hline$\quad$ Recurrence negative & 9 & 0 & 1 & 9 \\
\hline$\quad$ Unknown & & & 0 & \\
\hline$\quad$ Cocation & 32 & 7 & & \\
\hline$\quad$ Spinal & 8 & 1 & & \\
\hline
\end{tabular}

* Follow-up was available in 40 cases out of 49. 
diagnoses. At the time of initial diagnoses of the cases, immunomarkers such as epithelial membrane antigene (EMA), cytokeratin, vimentin, S100 and glial fibrillary acidic protein (GFAP) had been applied as well as the histological criteria of chordoma for the exclusion of other malignancies such as adenocarcinomas. PAS-Alcian Blue stain had been used for extracellular mucin identification. None of the cases had a concomitant malignancy.

Hyperchromasia was noted when more than $50 \%$ of the nuclei were intensely hyperchromatic.

Pleomorphism was noted when major cellular differences were detected in more than $50 \%$ of the tumor cells.

At least ten representative fields were analysed at high magnification (x400 objective) for determining mitosis.

All fields were examined to detect necrosis and any ratio of detectable necrosis was noted when seen.

This study was approved by the Ethics Committee of the relevant institute with the identifying code MARYC-2008-0213. An informed consent form regarding permission for clinical research was obtained from each patient.

\section{Immunohistochemistry}

Four-micrometer-thick sections were placed on 3-aminopropyletxylene-covered slides and deparaffinized at $60{ }^{\circ} \mathrm{C}$ for $1 \mathrm{~h}$. The slides were dewaxed in xylene and dehydrated in $96 \%$ alcohol. Sections were then immersed in $10 \mathrm{mmoV} \mathrm{l}$ citrate buffer ( $\mathrm{pH} \mathrm{6.0)}$ ) and microwaved for $20 \mathrm{~min}$ for antigen retrieval. Slides were cooled to room temperature and rinsed in phosphate-buffered saline (PBS). Endogenous peroxidase activity was blocked by further pretreatment with 3\% $\mathrm{H} 2 \mathrm{O} 2$ / distilled water for $20 \mathrm{~min}$ at room temperature. After a thorough washing in PBS, blocking solution (Lab Vision Co., Fremont, CA, USA) was applied to block nonspecific antibody binding.

The sections were then incubated with EGFR (SPRING, CA 94566, USA M3091: Ready to use), c-Met (Neomarkers, Fremont, CA 94539 USA RB-10286, 1:20 dilution) and c-ErbB2 (Neomarkers, Fremont, CA 94539 USA, 1:50 dilution) for $1 \mathrm{~h}$ at room temperature.

After a 10-min rinse in PBS, biotinylated goat antipolyvalent immunoglobulin (Lab Vision Co., Fremont, CA, 94539) for $20 \mathrm{~min}$, and in the streptavidin-biotin peroxidase complex for $20 \mathrm{~min}$ being applied at RT, $3,3^{1}$ diaminobenzidine chromogen was used for visualization of antigen-antibody binding. The sections were counterstained with hematoxylin, dehydrated, cleared in xylene and mounted on Entellan coverslips.

\section{Evaluation of Immunostaining}

Interpretation of c-ErbB2 staining was performed using the ASCO/CAP 2013 HER-2 Test Guideline in which membranous immunostaining of tumor that amounts to $10 \%$ of contiguous and homogeneous tumor cells was stated as positive. The EGFR staining was scored based on membranous staining intensity as $0=$ no staining; $1+=$ faint membranous staining in $>10 \%$ of tumor cells; $2+=$ moderate membranous staining; $3+=$ strong membranous staining. The c-Met staining was scored based on both membranous and cytoplasmic staining intensity as $0=$ no staining; $1+=$ faint staining in $>10 \%$ of tumor cells; $2+=$ moderate staining; $3+=$ strong staining. The scores were than categorized into two as $(0,1,2+)$ and $(3+)$. Background staining and staining in non-tumoral cells were considered as nonspecific staining and cases with this kind of staining pattern were interpreted as negative. The initial biopsy materials of the recurrent cases (cases with more than one biopsy specimen) were chosen for immunohistochemical evaluation.

\section{Statistical Analysis}

Statistical analysis was performed using the NCSS (Number Cruncher Statistical System) 2007 (NCSS, LLC Kaysville, Utah, USA, Hintze, J. (2008) programme.

Quantity statistics (Mean, Standard deviation, frequency) and qualitative data were analysed. Normally distributed parameters between groups were analyzed with Student's $t$ test; and the parameters that were not normally distributed between the groups were analyzed using the Mann-Whitney $\mathrm{U}$ test. Quantitative data were assessed with the chi-square test and Fisher's Exact chi-square test. Survival analysis was evaluated with Kaplan-Meier Survival Analysis excluding those lost to follow up or who died due to postoperative surgical complications. Log rank (mantel-Cox) analysis was used to compare the prognostic significance of the antibodies' expressions on survival. Results were evaluated using a 95\% confidence interval and significance was considered when the $\mathrm{p}$ value was lower than 0.05 .

\section{RESULTS}

\section{Patients and Follow up}

We included 49 patients with 31 males and 18 females in our study. The age of the cases differed between 5 and 83 years and the mean age at the time of diagnosis was $40.90 \pm 18.05$ years. Data from clinical follow-up of up to 168 months was available for 40 patients. The mean followup was $61.21 \pm 39.75$ months (range 1-168 months). Among the 40 patients 17 (42.5\%) had one or more recurrences. 
One patient had 5 recurrences, 1 patient had 3 recurrences, 2 patients had 2 recurrences while the other 13 patients had one recurrence. The mortality rate was $50 \%$ as 20 patients died. Among all cases, localization was cranial in 40 and spinal in 9 patients.

\section{Histopathological Evaluation}

According to the WHO classification; 40 tumors were of the conventional type (Figure 1), 8 were chondroid (Figure 2) and 1 was dedifferentiated (Figure 3 ). The dedifferentiated histology was present in the initial biopsy of the patient. The case with dedifferetiated histology had been nonrecurrent after being followed for 84 months.

On histopathological examination, mitosis was found in 2 cases, necrosis in 6 cases (Figure 4), pleomorphism in 4 cases, and hyperchromatic nuclei in 15 cases.

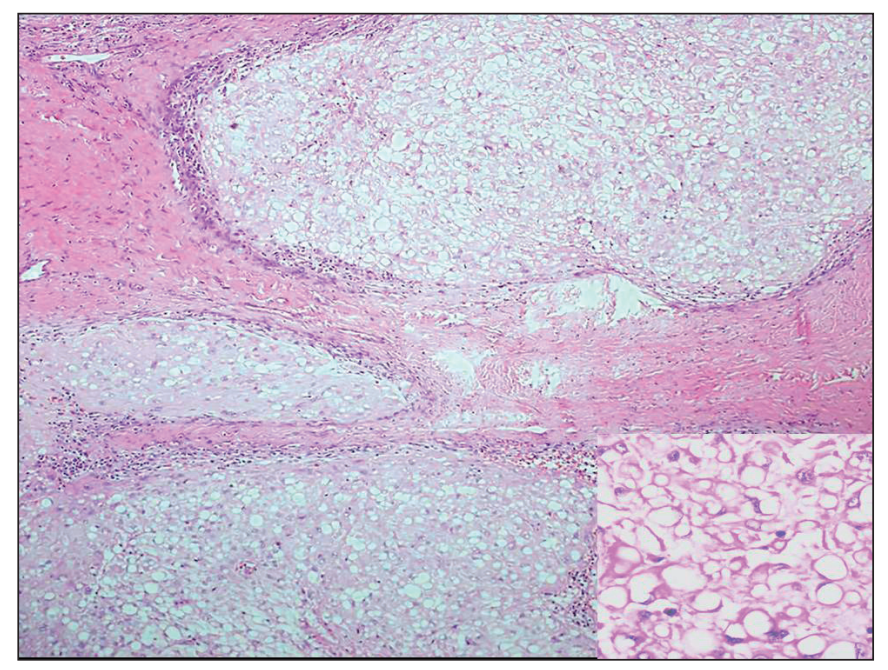

Figure 1: Conventional chordoma with nodular growth pattern and physalipherous cells (inset) (H\&E; x100, H\&E; x400).

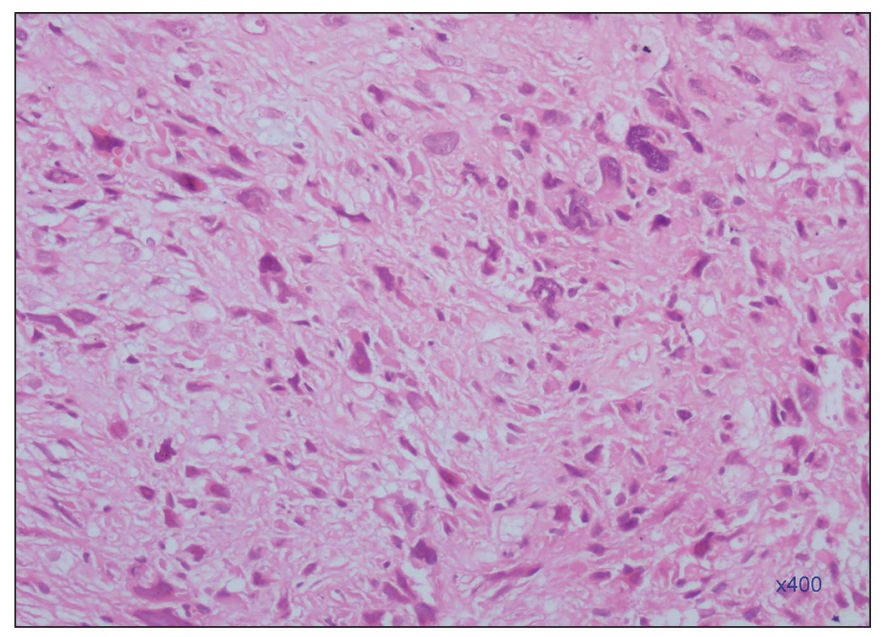

Figure 3: Dedifferentiated chordoma (H\&E; x400).
Two cases showing mitotic activity were dedifferentiated and conventional chordomas respectively. Mitotic activity had been $4 / 10 \mathrm{HPF}$ (High power field) in the dedifferentiated variant and 2/10 HPF in the conventional variant.

Three of 6 cases with necrosis were conventional chordomas while two were chondroid and the remaining one was dedifferentiated chordoma. The highest extension ratio of necrosis (necrotic area/total tumor area) was $50 \%$ while the lowest ratio was $10 \%$.

Two of the 4 cases showing pleomorphism had chondroid morphology while the others had been dedifferentiated and conventional chordomas. The case with dedifferentiated histology revealed marked pleomorphism and atypical features such as sarcomatoid areas, high nuclear/ cytoplasmic ratios and irregular nuclear membranes. Mild pleomorphism was present in the other three cases.

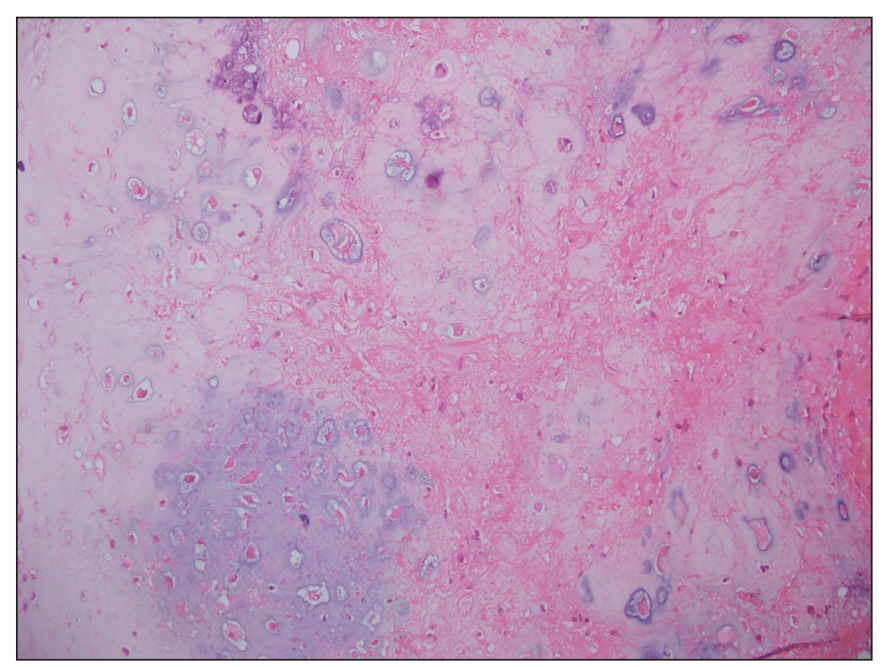

Figure 2: Chondroid chordoma (H\&E; x200).

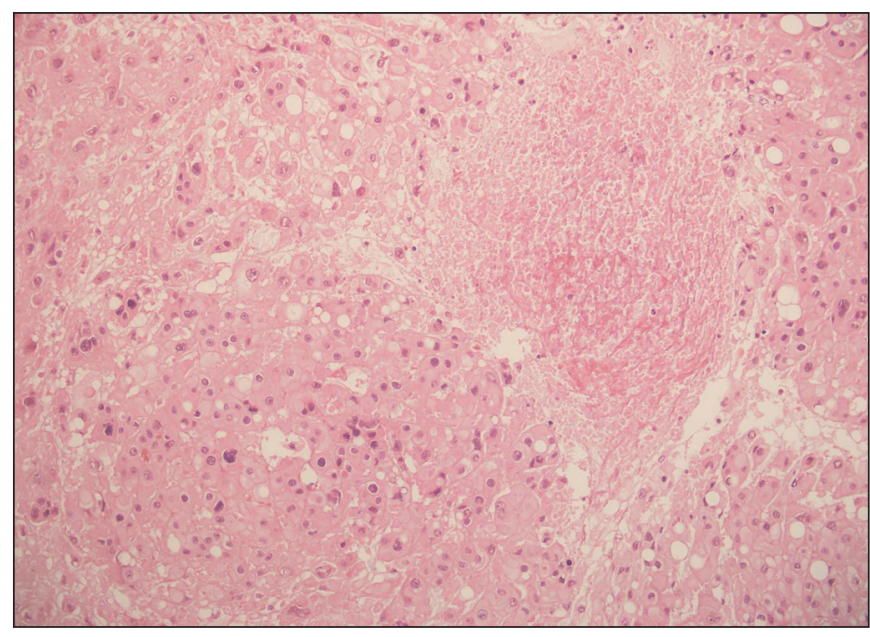

Figure 4: Necrosis (H\&E; $\mathrm{x} 200)$. 
Table II: Histopathological parameters of the cases

\begin{tabular}{lcccc}
\hline Histopathological parameters & \multicolumn{2}{c}{ Present } & \multicolumn{2}{c}{ Absent } \\
\cline { 2 - 5 } Total n: 49 & n & \% & n & \% \\
\hline Mitosis & 2 & 4 & 47 & 96 \\
\hline Necrosis & 6 & 12 & 43 & 88 \\
\hline Pleomorphism & 4 & 8 & 45 & 92 \\
\hline Hyperchromatic nuclei & 15 & 31 & 34 & 69 \\
\hline Mucin & 49 & 100 & 0 & 0 \\
\hline Apoptosis & 0 & 0 & 49 & 100 \\
\hline
\end{tabular}

Apoptosis was not observed in any of the cases and all cases showed mucin deposition (Table II).

The only significant statistical correlation among histopathological parameters was between necrosis and survival rates (Table III, Figure 5). Survival rates were lower in cases with necrosis $(\mathrm{p}<0.01)$. No correlation was detected between histological features and prognosis (recurrence and overall survival).

\section{Expression of EGFR, c-Met and c-ErbB2}

Immunohisto-chemical evaluation revealed that $77.5 \%$ of the cases (38/49) were intensely positive (3+) by EGFR (Figure 6) and 12.2\% (6/49) by c-Met (Figure 7). Two cases were negative by EGFR and 34 were negative by c-Met. None of the cases stained by c-ErbB2. The only significant correlation was found between c-Met expression and recurrence. Recurrence rates were higher in c-Met intensely positive cases $(\mathrm{p}<0.05)($ Table IV). No significant correlation was found between immunoexpression profiles and clinicopathological parameters including age, sex, localization of tumor, histological type and the histopathological properties of the tumor.

\section{DISCUSSION}

Although studies based on the correlation of prognosis and histopathological parameters such as mitosis, apoptosis and extracellular mucin in chordoma are present in the literature, no definite evidence-based histopathological adverse prognostic factor predicting recurrence has been found $(22,23)$. Chordomas are believed to originate from mesenchymal tissue; however they have recently been considered to have dual epithelial and mesenchymal differentiation (2). Receptor tyrosine kinases such as EGFR, c-Met ve c-ErbB2 are implicated in the pathogenesis of various epithelial tumors. The molecular-targeted therapies have been recently administered to patients whose tumors have tyrosine kinase activity $(15,19,21)$. In our study, a cranial localization (81.6\% of all cases) was the most frequent site. This finding contradicts the previous studies

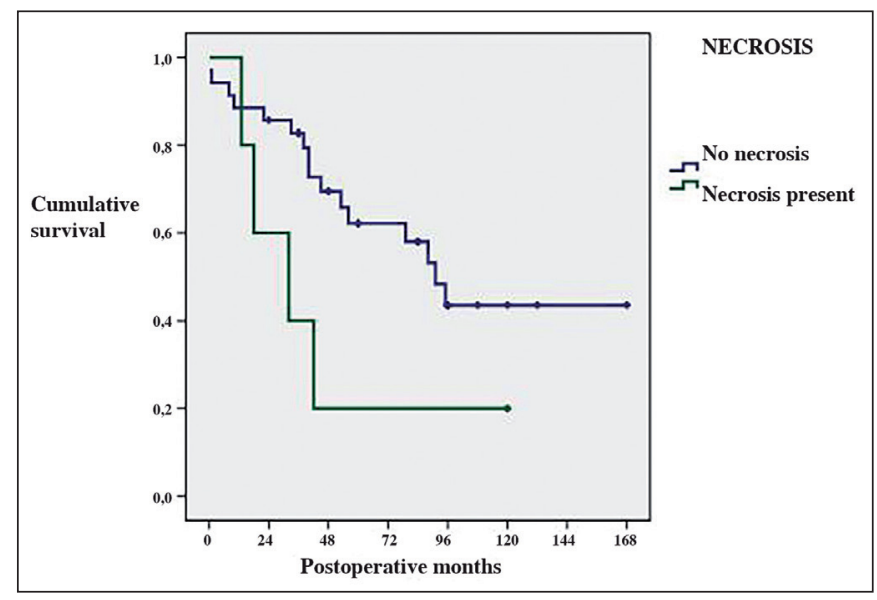

Figure 5: The Kaplan-Meier survival curves of two groups of cases according to presence of necrosis.

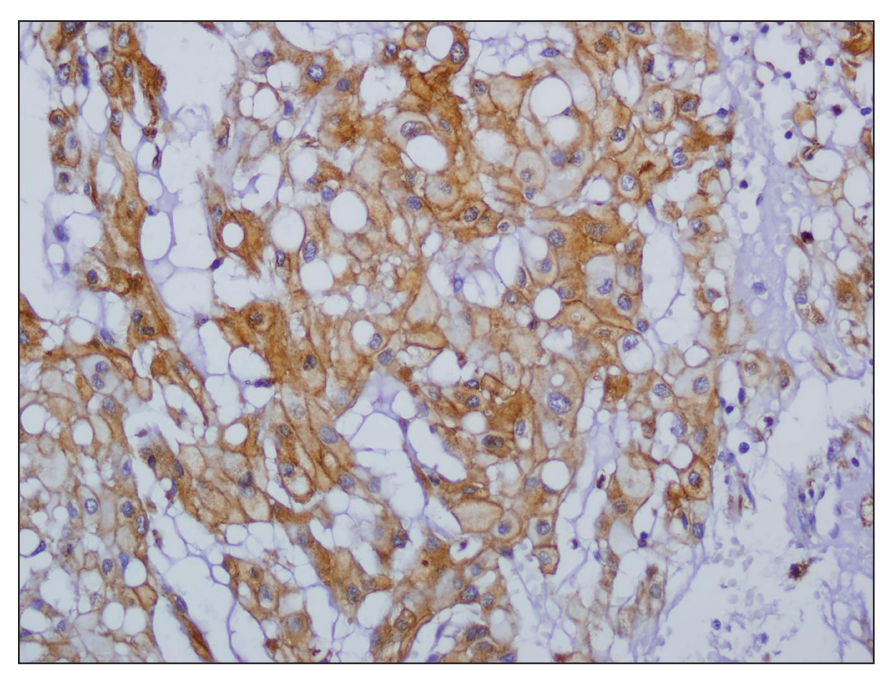

Figure 6: 3+ EGFR immunoexpression in a case (EGFR; x200).

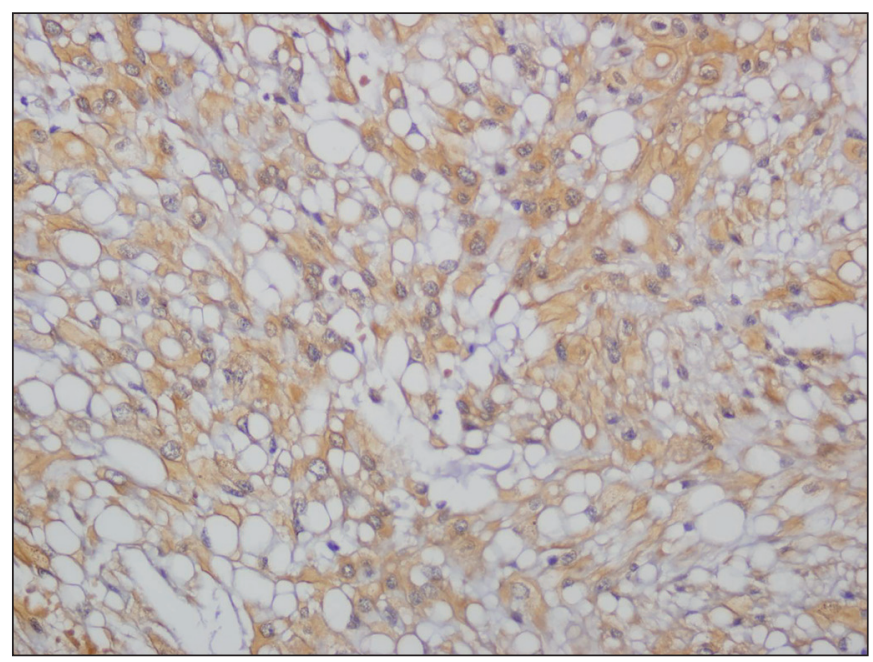

Figure 7: 3+ c-Met immunoexpression in a case (c-Met; x200). 
Table III: Necrosis and survival analysis

\begin{tabular}{|c|c|c|c|c|c|c|c|}
\hline \multirow{2}{*}{ Necrosis } & \multirow{2}{*}{ Total } & \multirow{2}{*}{ Exitus } & \multirow{2}{*}{ Alive } & \multirow{2}{*}{ Survival Rate (\%) } & \multicolumn{2}{|c|}{ Cummulative Survival } & \multirow{2}{*}{ Mean survival time (months) } \\
\hline & & & & & Mean (\%) & Standard Error (\%) & \\
\hline Present & 5 & 4 & 1 & 20 & 20,0 & 17.9 & $45.0 \pm 17.38$ \\
\hline Absent & 35 & 16 & 19 & 54.3 & 43.5 & 10 & $101.21 \pm 11.76$ \\
\hline
\end{tabular}

Kaplan-Meier Analysis ${ }^{*} \mathrm{p}<0.05$

Table IV: Correlation of EGFR, c-Met and c-ErbB2 profiles with recurrence

\begin{tabular}{|c|c|c|c|c|c|}
\hline & Total (n:49) & Follow-up Available (n:40) & Recurrent (n:17) & Nonrecurrent (n:23) & p-Value \\
\hline \multicolumn{6}{|l|}{ EGFR } \\
\hline $0-1-2$ & 11 & 9 & 3 & 6 & \multirow{2}{*}{0.294} \\
\hline 3 & 38 & 31 & 14 & 17 & \\
\hline \multicolumn{6}{|l|}{ c-Met } \\
\hline $0-1-2$ & 43 & 36 & 13 & 23 & \multirow{2}{*}{$0.026^{*}$} \\
\hline 3 & 6 & 4 & 4 & 0 & \\
\hline \multicolumn{6}{|l|}{ c-ErbB2 } \\
\hline Total & 49 & 40 & 17 & 23 & \multirow[t]{2}{*}{$>1$} \\
\hline $0-1-2-3$ & 0 & 0 & 0 & 0 & \\
\hline
\end{tabular}

*Mann-Whitney U test

in which the sacrococcyx is presented as the most frequent site $(24,25)$. The reason of this contradiction may be the sample size of our study.

Chordoma predominantly affects men aged $40-50$ years $(5$, 24-26). In our study, the mean age of the patients was $40.9 \pm$ 18.05 years and $63.3 \%$ of the patients were male. In a study of 51 cases, survival rates were better in patients younger than 40 years regardless of the tumor's histopathological features, and age was defined as an independent factor (27). O'Connel et al. proposed female gender as a poor prognostic indicator in their study of 62 patients (28).

The most effective treatment for chordoma is still surgery. Complete resection or gross total resection chance depends on the localization of the tumor. Residual chordoma deeply infiltrating the surrounding tissues mainly account for the local recurrences. For complete tumor removal, a radical wide posterior surgical margin should be employed while dealing with sacrococcygeal cases (26). McGirt et al. assessed 67 sacral and 47 mobile spine chordoma patients who had been surgically treated and revealed that sacral localization was associated with poor survival in chordoma (29). In another study based on 29 cases, vertebral localization appeared to be a significant risk factor for recurrence (30). In a study by Fasig et al., no correlation was found between tumor localization and recurrence in 20 chordoma cases (16). We have not found a specific correlation between localization and recurrence in our study.
Eight cases $(16.3 \%)$ in our study were diagnosed as chondroid chordoma. This finding is compatible with the most comprehensive study of chordoma in which 22 of 155 (14\%) cases were diagnosed as chondroid chordoma (6).

In a study based on chondroid chordoma, survival rates were found to be better in chondroid chordoma compared with classical chordoma (6). In another study, no specific correlation was found between the histological type of chordoma and prognosis (28). In our study, we have not found a statistically significant correlation between the histological type and prognosis of chordoma.

Necrosis and survival rates have been found to be correlated with each other. Survival rates are worse in the cases in which necrosis is reported. This result is consistent with the two other studies in the chordoma literature $(28,31)$. However, there are other studies claiming that necrosis has no role in chordoma prognosis $(22,32)$.

No statistical correlation was found between the other parameters (mitosis, pleomorphism, presence of mucin) and prognosis.

In the study of Bjornsson et al. chondroid differentiation and cellular atypia were investigated and these parameters were not correlated with the biological behaviour of chordoma (32). Histopathological features such as hypercellularity, pleomorphism, apoptosis, necrosis and proliferative index by $\mathrm{Ki}-67$ antibody were assessed in 
another study. Tumor growth was found to be increased in cases showing high mitotic ratios and a proliferative index more than $6 \%$ (22). Mitosis was reported only in two cases in our study. No statistically signficant correlation was shown between cases with mitotic activity and prognosis. The controversial results of the studies in the literature can be explained by the low number of the cases due to the rarity of chordoma.

A limitation of our study is the correlation of survival and prognosis with the proliferative index shown by Ki-67 antibody. We have used the residual tissues in the paraffin blocks of the patients after the routine reporting process. During the reporting process, almost all of the cases were evaluated not only by H\&E stain but also immunohistochemical stains such as EMA, S100, GFAP, vimentin and $\mathrm{CK}$. The majority of cases included in our study have been intracranial chordoma cases with limited tissue samples with volumes lower than $1 \mathrm{~cm}^{3}$. After the preparation of the routine $\mathrm{H} \& \mathrm{E}$ stain, histochemical PAS-Alcian Blue stain, sections were taken for the immunohistochemical analysis of three receptor tyrosine kinases. Immunohistochemical Ki-67 application was available in 10 cases and the proliferative activities were less than $1 \%$ in 6 cases, $2 \%$ in two cases, $3 \%$ in one case and $5 \%$ in one case.

Immunohistochemical staining revealed that $77.6 \%$ of the cases were intensely positive (3+) by EGFR and $12.2 \%$ by c-Met. None of the cases stained by c-ErbB2. A statistically significant correlation was found between c-Met expression and recurrence. Recurrence rates were higher in patients with high levels of c-Met expression. A positive correlation was determined between extension of staining by EGFR and c-Met. EGFR positive cases showed significant c-Met immunoexpression $(\mathrm{p}<0,01)$.

Weinberger et al. examined the expression of c-Met, c-Erb-b2 (HER2/neu), and epidermal growth factor (EGFR) in a cohort of 12 chordomas and found that all chordomas had positive expressions of both the hepatocyte growth factor/scatter factor receptor and EGFR. c-ErbB2 expression was also detected in 7 out of 12 cases. Another outcome of this study was the positive correlation between EGFR and c-Met expressions. Chordoma tumors that had high c-Met expression were also likely to have high EGFR expression (17).

In a study by Akhavan-Sigari at al. using immunohistochemical techniques, the authors investigated Plateletderived growth factor receptor- $\alpha$ (PDGFR $\alpha$ ), EGFR and c-Met expression in 52 primary lesions (33). PDGFR- $\alpha$,
EGFR and c-Met were found to be expressed in $75.0 \%$, $83 \%$ and $77 \%$ of primary lesions. In the subsequent study of Akhavan-Sigari et al. positive immunostaining with EGFR and c-Met was detected in $92 \%$ and $100 \%$ of 145 tumor specimens (34). Fasig et al. studied receptor tyrosine kinases in 21 chordoma cases and found PDGFR-b, EGFR, KIT and c-ErbB2 expressions in 100\%, 67\%, 33\% and 0\% of cases, respectively (16). Naka et al. investigated HGF/cMet expression in 46 primary and 25 recurrent lesions and found c-Met expression in $70.0 \%$ of primary and $88.0 \%$ of recurrent lesions (35). EGFR expression has been reported in $67-100 \%$ and c-Met expression varies between $70 \%$ and $100 \%$ in these corresponding studies. In our study, EGFR and c-Met expression rates were $77.6 \%$ and $12.2 \%$. The c-Met expression rate is lower than the rates given in the studies mentioned in the literature. This discrepancy with the literature could be associated with the clone of the antibody used in our study which differed from the clone and brand of c-Met antibody that had been used in the regarding studies. This discordance could also be explained by the sample size. Further molecular research as well as immunohistochemical studies with a larger number of cases are indicated in order to analyse the expression rate of c-Met in chordoma. Peghini et al. demonstrated positive correlation with EGFR and c-Met staining in gastrinomas and the staining was also related with the aggressive behaviour of the tumors (36).

Pharmacological inhibition of EGFR kinase has no impact on the blockage of c-Met activity, however this inhibition abolishes the proliferative effect after HGF binds to its receptors (37). Common downstream pathways between EGFR, HGF and c-Met may explain this mechanism. A study of 21 chordoma cases analysing the genes in the downstream pathways of EGFR including p44/42 MAPK, Akt, STAT3 has revealed that $67 \%$ of the cases were reactant by EGFR while the phosphorylated forms of p44/42 MAPK, Akt, STAT3 were expressed in $86 \%, 76 \%$, and $\% 67$ respectively. The high expression profiles of these genes strongly indicate a role for thyrosine kinase activity in the pathogenesis of chordoma (16). Microarray studies analysing multiple genes are required to examine the exact mechanism of EGFR, cMet and c-ErbB2 in chordoma.

The c-Met protooncogene is located in chromosome $7 \mathrm{q} 31.21$. Scheil et al. showed $7 \mathrm{q}$ gain in $69 \%$ of chordomas (38). c-Met expression via $7 \mathrm{q}$ amplification may be a boarding step of chordoma progression. c-Met and HGF are reported to be implicated in chordoma etiology and metastatic progression (39). 
Recurrence rates were higher in cases with intensely positive c-Met staining. All of the 4 cases with high c-Met expression showed recurrences. One of them showed two recurrences and three showed a single recurrence. Localization was cranial in all cases and gross total resection was performed in each case. Two of the cases died at the 33rd and 45th months while the others were alive. Follow-up periods for the latter were 96 and 168 months after surgery. Three of these cases were conventional while one was chondroid. Hyperchromatic nuclei were evident in a single case and the others did not show any atypical histological feature at the time of initial biopsies.

There are studies in the literature revealing the correlation of c-Met staining and poor prognosis in glioblastoma, bladder, cervix, lung and liver neoplasms and multiple myeloma (40). In the study of Akhavan-Sigari et al., recurrent chordoma lesions with higher c-Met expression were found to be associated with significantly better prognosis than those with lower c-Met expression. Those patients with lower EGFR expression were found to have significantly better prognosis than those with higher EGFR expression (33). However, in our study, recurrence rates were high in intensely c-Met positive cases. This finding could also be explained by the limited sample size of the studies. Therefore meta-analysis reports should be prepared to obtain more reliable data about the correlation between the histological features and immunostaining profiles of chordoma and prognosis.

None of our cases showed immunoreactivity by c-ErbB2. Conflicting results are present comparing the two other studies investigating c-ErbB2 in chordoma. Weinberger et al. claim that c-ErbB2 immunoreactivity is seen in 7 cases out of 11 (17) while in the study of Fasig et al no reactivity by c-ErbB2 is demonstrated although two different clones of this antibody being used (16). Our results are consistent with the study of Fasig et al.

Complementary studies such as flourescence in situ hybridization and Western Blot analysis with more cases are needed to argue whether c-ErbB2 has a role in the pathogenesis of chordoma.

Because of the aggressive potential and poor prognosis of chordoma, new therapeutical strategies should be investigated. Hof et al. presented a case of chordoma under a combination regimen including cetuximab and gefitinib who showed partial regression in pulmonary metastasis (18). c-Met expression could be used as an indicator of recurrence risk, as a target for therapeutic agents to decrease recurrence, and for the prediction of prognosis.
In conclusion, although a high percentage of the cases showed EGFR overexpression, no statistical correlation was found between EGFR immunoreactivity and survival. Further studies are required in order to determine the population of patients with chordoma who are likely to benefit from EGFR inhibitors. Recurrences were more common in cases with c-Met overxpression than ones with lower c-Met expression levels. c-Met inhibitors may play a role in controlling the disease in the patients with c-Met overexpression.

\section{CONFLICT of INTEREST}

The authors declare that there are no conflicts of interest and no relevant financial interests in this manuscript.

\section{REFERENCES}

1. McMaster ML, Goldstein AM, Bromley CM, Ishibe N, Parry DM. Chordoma: Incidence and survival patterns in the United States, 1973-1995. Cancer Causes Control. 2001;12:1-11.

2. Chugh R, Tawbi H, Lucas DR, Biermann JS, Schuetze SM, Baker LH. Chordoma: The nonsarcoma primary bone tumor. Oncologist. 2007;12:1344-50.

3. Pamir MN, Kiliç T, Türe U, Ozek MM. Multimodality management of 26 skull-base chordomas with 4-year mean follow-up: Experience at a single institution. Acta Neurochir (Wien). 2004;146:343-54.

4. Baratti D, Gronchi A, Pennacchioli E, Lozza L, Colecchia M, Fiore M, Santinami M. Chordoma: Natural history and results in 28 patients treated at a single institution. Ann Surg Oncol. 2003;10:291-6.

5. Gentil F, Coley BL. Sacrococcygeal chordoma. Ann Surg. 1948;127:432-55.

6. Heffelfinger MJ, Dahlin DC, MacCarty CS, Beabout JW. Chordomas and cartilaginous tumors at the skull base. Cancer. 1973;32:410-20.

7. Fleming GF, Heimann PS, Stephens JK, Simon MA, Ferguson MK, Benjamin RS, Samuels BL. Dedifferentiated chordoma. Response to aggressive chemotherapy in two cases. Cancer. 1993;72:714-8.

8. Mitsudomi T, Yatabe Y. Epidermal growth factor receptor in relation to tumor development: EGFR gene and cancer. FEBS J. 2010;277:301-8

9. Yarden Y. The EGFR family and its ligands in human cancer. signalling mechanisms and therapeutic opportunities. Eur J Cancer. 2001;37 Suppl 4:S3-8.

10. Wolff AC, Hammond ME, Hicks DG, Dowsett M, McShane LM Allison KH, Allred DC, Bartlett JM, Bilous M, Fitzgibbons P, Hanna W, Jenkins RB, Mangu PB, Paik S, Perez EA, Press MF, Spears PA, Vance GH, Viale G, Hayes DF; American Society of Clinical Oncology; College of American Pathologists. Recommendations for human epidermal growth factor receptor 2 testing in breast cancer: American Society of Clinical Oncology/ College of American Pathologists clinical practice guideline update. J Clin Oncol. 2013;31:3997-4013. 
11. Boon EM, van der Neut R, van de Wetering M, Clevers H, Pals ST. Wnt signaling regulates expression of the receptor tyrosine kinase met in colorectal cancer. Cancer Res. 2002;62:5126-8.

12. Weidner KM, Arakaki N, Hartmann G, Vandekerckhove J, Weingart S, Rieder H, Fonatsch C, Tsubouchi H, Hishida T, Daikuhara Y. Evidence for the identity of human scatter factor and human hepatocyte growth factor. Proc Natl Acad Sci U S A. 1991;88:7001-5.

13. Basilico C, Arnesano A, Galluzzo M, Comoglio PM, Michieli P. A high affinity hepatocyte growth factor-binding site in the immunoglobulin-like region of Met. J Biol Chem. 2008;283:21267-77.

14. Blume-Jensen $P$, Hunter T. Oncogenic kinase signalling. Nature .2001;411:355-65.

15. Pegram MD, Konecny G, Slamon DJ. The molecular and cellular biology of HER2/neu gene amplification/overexpression and the clinical development of herceptin (trastuzumab) therapy for breast cancer. Cancer Treat Res. 2000;103:57-75.

16. Fasig JH, Dupont WD, LaFleur BJ, Olson SJ, Cates JM. Immunohistochemical analysis of receptor tyrosine kinase signal transduction activity in chordoma. Neuropathol Appl Neurobiol. 2008;34:95-104

17. Weinberger PM, Yu Z, Kowalski D, Joe J, Manger P, Psyrri A, Sasaki CT. Differential expression of epidermal growth factor receptor, c-Met, and HER2/neu in chordoma compared with 17 other malignancies. Arch Otolaryngol Head Neck Surg. 2005; 131:707-11.

18. Hof H, Welzel T, Debus J. Effectiveness of cetuximab/gefitinib in the therapy of a sacral chordoma. Onkologie. 2006;29:572-4.

19. Schlessinger J. Cell signaling by receptor tyrosine kinases. Cell. 2000;103:211-25.

20. Ruco LP, Stoppacciaro A, Ballarini F, Prat M, Scarpino S. Met protein and hepatocyte growth factor (HGF) in papillary carcinoma of the thyroid: Evidence for a pathogenetic role in tumourigenesis. J Pathol. 2001;194:4-8.

21. Hayes DF, Thor AD. c-erbB-2 in breast cancer: Development of a clinically useful marker. Semin Oncol. 2002;29:231-45.

22. Holton JL, Steel T, Luxsuwong M, Crockard HA, Revesz T. Skull base chordomas: Correlation of tumour doubling time with age, mitosis and Ki67 proliferation index. Neuropathol Appl Neurobiol. 2000;26:497-503.

23. Kaiser TE, Pritchard DJ, Unni KK. Clinicopathologic study of sacrococcygeal chordoma. Cancer. 1984;53:2574-8.

24. Higinbotham NL, Phillips RF, Farr HW, Hustu HO. Chordoma. Thirty-five-year study at Memorial Hospital. Cancer. 1967;20:1841-50.

25. Dahlin DC, Maccarty CS. Chordoma. Cancer. 1952;5:1170-8.

26. Yonemoto T, Tatezaki S, Takenouchi T, Ishii T, Satoh T, Moriya $\mathrm{H}$. The surgical management of sacrococcygeal chordoma. Cancer. 1999;85:878-83.
27. Mitchell A, Scheithauer BW, Unni KK, Forsyth PJ, Wold LE, McGivney DJ. Chordoma and chondroid neoplasms of the spheno-occiput. An immunohistochemical study of 41 cases with prognostic and nosologic implications. Cancer. 1993;72:2943-9.

28. O’Connell JX, Renard LG, Liebsch NJ, Efird JT, Munzenrider JE, Rosenberg AE. Base of skull chordoma. A correlative study of histologic and clinical features of 62 cases. Cancer. 1994;74: 2261-7

29. McGirt MJ, Gokaslan ZL, Chaichana KL. Preoperative grading scale to predict survival in patients undergoing resection of malignant primary osseous spinal neoplasms. Spine J. 2011;11:190-6.

30. Sarsık B, Doganavsargil B, Başdemir G, Zileli M, Sabah D, Öztop F. Chordomas: Is it possible to predict recurrence? Turk Patoloji Derg. 2009;25:27-34.

31. Bergh P, Kindblom LG, Gunterberg B, Remotti F, Ryd W, MeisKindblom JM. Prognostic factors in chordoma of the sacrum and mobile spine: A study of 39 patients. Cancer. 2000; 88: 2122-34.

32. Bjornsson J, Wold LE, Ebersold MJ, Laws ER. Chordoma of the mobile spine. A clinicopathologic analysis of 40 patients. Cancer. 1993;71:735-40.

33. Akhavan-Sigari R, Gaab MR, Rohde V, Abili M, Ostertag H. Expression of PDGFR-alpha, EGFR and c-MET in spinal chordoma: A series of 52 patients. Anticancer Res. 2014;34:62330 .

34. Akhavan-Sigari R, Abili M, Gaab MR, Rohde V, Zafar N, Emami $\mathrm{P}$, Ostertag $\mathrm{H}$. Immunohistochemical expression of receptor tyrosine kinase PDGFR-á, c-Met, and EGFR in skull base chordoma. Neurosurg Rev. 2015;38:89-98; discussion 98-9.

35. Naka T, Kuester D, Boltze C, Scheil-Bertram S, Samii A, Herold C, Ostertag H, Krueger S, Roessner A. Expression of hepatocyte growth factor and c-MET in skull base chordoma. Cancer. 2008;112:104-10.

36. Peghini PL, Iwamoto M, Raffeld M, Chen YJ, Goebel SU, Serrano J. Overexpression of epidermal growth factor and hepatocyte growth factor receptors in a proportion of gastrinomas correlates with aggressive growth and lower curability. Clin Cancer Res. 2002;8:2273-85.

37. Scheving LA, Stevenson MC, Taylormoore JM, Traxler P, Russell WE. Integral role of the EGF receptor in HGF-mediated hepatocyte proliferation. Biochem Biophys Res Commun. 2002;290:197-203.

38. Scheil S, Brüderlein S, Liehr T, Starke H, Herms J, Schulte M. Genome-wide analysis of sixteen chordomas by comparative genomic hybridization and cytogenetics of the first human chordoma cell line, U-CH1. Genes Chromosomes Cancer. 2001;32:203-11.

39. Ostroumov E, Hunter CJ. Identifying mechanisms for therapeutic intervention in chordoma: c-Met oncoprotein. Spine (Phila Pa 1976). 2008;33:2774-80.

40. Birchmeier C, Brohmann H. Genes that control the development of migrating muscle precursor cells. Curr Opin Cell Biol. 2000;12:725-30. 